\section{Multifocal Osteomyelitis in a Neonate, an Overview of Diagnosis and Treatment}

\section{Somford MP ${ }^{1 *}$, Huibers $\mathrm{MHW}^{2}$, Schuppen $\mathrm{J}^{3}$, Struijs PAA ${ }^{4}$ and van Lee $\mathbf{R}^{2}$}

${ }^{1}$ Department of Orthopedic Surgery, Medisch Spectrum Twente, Enschede, Netherlands

${ }^{2}$ Department of Neonatology, Academic Medical Center, Amsterdam, Netherlands

${ }^{3}$ Department of Radiology, Academic Medical Center, Amsterdam, Netherlands

${ }^{4}$ Department of Orthopedic Surgery, Academic Medical Center, Amsterdam, Netherlands

\begin{abstract}
We present a case of multifocal osteomyelitis and septic arthritis in a 26 weeks premature neonate. A female newborn child presented with sepsis-like illness systemically as well as multiple swelling of the bones and joints. After clinical suspicion of osteomyelitis, the diagnosis was made with the help of blood culture and imaging studies such as ultrasound, plain radiographs and MRI. The child underwent multiple surgeries for drainage of the abscesses and infected joints. Sensitive antibiotics were continued perioperative as well as postoperative for 6 weeks after the last surgery. Inflammatory marker and MRI were used to follow prognosis. The child recovered well without notable short term sequellae at 18 months follow up.
\end{abstract}

\section{Introduction}

Osteomyelitis with arthritis in the neonatal period is a rare but severe condition. The incidence is approximately $0.5 \%$ of all neonatal admissions. Misdiagnosis is commonly due to unclear signs and symptoms. Delayed treatment can worsen outcome leading to permanent sequellae [1]. We present a unique case of multi-focal Septic Arthritis (SA) and acute Osteomyelitis (OM) due to a Staphylococcus aureus infection of unknown origin.

\section{Case Report}

A negroid neonate was born at 26 weeks of gestation as the first child of both parents. Besides prematurely ruptured membranes at

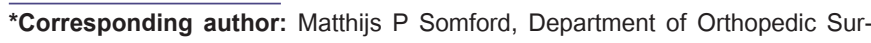
gery, Medisch Spectrum Twente, Enschede, Netherlands, Tel: +31 624881380; E-mail: Mp_Somford@hotmail.com

Citation: Somford MP, Huibers MHW, Schuppen J, Struijs PAA, van Lee R (2015) Multifocal Osteomyelitis in a Neonate, an Overview of Diagnosis and Treatment. J Orthop Res Physiother 1: 015.

Received: August 06, 2015; Accepted: October 19, 2015; Published: November 02,2015
25 weeks of gestation no other complications during pregnancy were reported. The girl was born after a spontaneous delivery with a birth weight of 795 gram (p20-p50). Apgar-scores were 3 and 4 after, respectively, 1 and 5 minutes. Antibiotics were administered directly after birth because of a high risk of infection. Skin, nose and throat cultures showed a group B Streptococcus, however blood cultures remained negative 48 hours after birth and consequently antibiotics were stopped. After three weeks, the girl developed a sepsis-like illness with respiratory failure as the leading symptom. During physical examination a redness of the proximal part of the right upper leg $(1-1 \mathrm{~cm})$, a swollen metatarsal 1 region of the right foot, a vesicle on the right ankle and swelling of left elbow were noted. The infant was started on flucloxacillin and gentamycin after cultures were taken from the skin of the right foot, blood, urine and spinal fluid. Skin and blood cultures were positive for Staphylococcus aureus with good sensitivity to flucloxacillin; no resistance against antibiotics was tested. Blood tests showed a C-reactive protein of $305 \mathrm{mg} / \mathrm{l}$ (normal $<1$ $\mathrm{mg} / \mathrm{l}$ ) and high levels of white blood cell count $41.9 \times 10^{*} 9 / 1$ (normal $\left.6.0-17.0 \times 10^{*} 9 \backslash 1\right)$. Antibiotic treatment was continued with Flucloxacillin (150 mg/kg in 4 doses). However, swelling of the right ankle and foot as well as the left elbow persisted after 4 days of treatment. An ultrasound of the foot and elbow showed subcutaneous infiltration and a fluctuating subcutaneous fluid collection with air configurations. This collection was continuous with the talonavicular joint. The navicular and cuboid bone showed a subperiostal fluid collection and possible air inside the bone, which was confirmed with conventional imaging (Figure 1). Ultrasound of the left elbow showed subcutaneous infiltration with a subperiostal fluid collection in the distal humeral and proximal radial bone. Conventional imaging confirmed a lucent aspect of the distal humerus and proximal radial bone with soft tissue infiltration. This was in keeping with acute osteomyelitis (Figure 1). An MR was performed which confirmed osteomyelitis with a soft tissue and subperiosteal abscess of both the right ankle and the left elbow joint (Figure 2). The orthopedic surgeon was consulted and surgery was performed immediately. During surgery pus was evacuated from the midfoot, the left distal humerus and elbow and the left radius and ulna. The pus was located both in the soft tissues and subperiosteally. All cultures were positive for Staphylococcus aureus and sensitive for flucloxacilline in vitro. Cardiological evaluation showed no signs of endocarditis. After 3-4 days swelling of left elbow and right foot persisted despite daily rinsing using small infusion systems as drains. A total body MR scan was performed and abscesses were located in the hips, knees, the left elbow and a large fluid collection in the right gluteus maximus muscle (Figure 2). Again exploratory surgery was performed. Pus was evacuated from the right knee and the right gluteal area. Both hips and the left knee only contained turbid fluid. Before surgery a new swelling of the wrist was seen. Exploration and evacuated during the surgery showed pus as well. All joint cultures taken during these cultures were negative for bacteria including Staphylococcus aureus after ten days. Because of continuing progression of infection during the use of flucloxacilline for 5 days, rifampicin $(20 \mathrm{mg} / \mathrm{kg})$ was started. Rifampicin was given for a total of 7 days and discontinued when swelling of the affected joints was markedly reduced. Unfortunately 2 days after 
discontinuation of rifampicin, a new swelling was seen on the right ankle and left elbow. A new MR was performed and again we found signs of osteomyelitis of the left distal humerus with abscess formation and accompanying osteomyelitis of the radial head and arthritis of the elbow joint, osteomyelitis of the right distal humerus on the lateral side without abscess, a large hematoma in the right gluteus maximus, possibly infected, and a hypointense abnormality centrally in the right talus. Another surgical rinsing was performed of the left distal humerus and the right gluteal region. No pus was evacuated, only turbid fluid. After surgery some swelling remained, but no other clinical signs were noted. Rifampicin was re-started and the patient was closely observed. The swelling on the right ankle and left elbow subsided over time. Rifampicin was discontinued after 5 days when cultures of joint spill and blood remained negative. Flucloxacilline $(150 \mathrm{mg} / \mathrm{kg})$ was given continuously during 6 weeks after the third operation. C-reactive protein and the white blood cell count normalized after 4 weeks. Immunological disorders were ruled out by a normal count of total immunoglobulin $(2.1 \mathrm{~g} / \mathrm{l})$ and normal specific granulocyte function test. Follow-up at the age of 18 months revealed no permanent sequellae, development of the motor functions was normal.

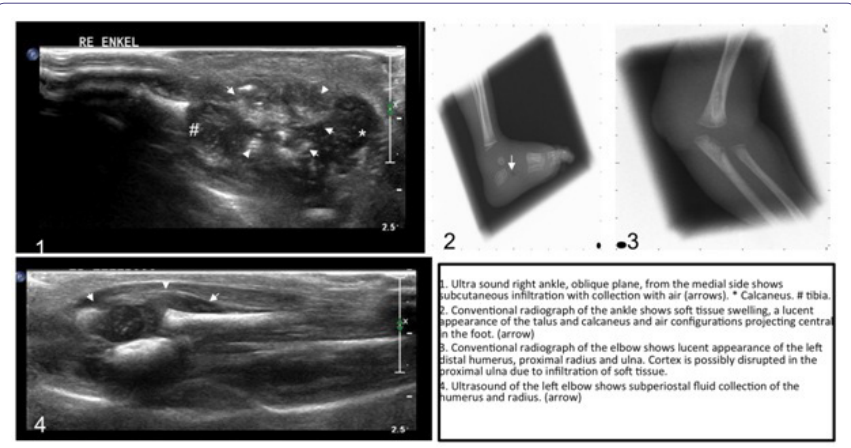

Figure 1: 1 - Ultra sound right ankle, oblique plane, from the medial side shows subcutaneous infiltration with collection with air (arrows) * - calcaneus, \# - tibia.

2 - Conventional radiograph of the ankle shows soft tissue swelling, a lucent aspect of the talus and calcaneus and air configurations projecting central in the foot (arrow).

3 - Conventional radiograph of the elbow shows lucent aspect of the left humerus, proximal radius and ulna. Cortex is possibly disrupted in the proximal ulna due to infiltration of soft tissue.

4 - Ultrasound of the left elbow shows subperiostal fluid collection of the humerus and radius (arrow).

\section{Discussion}

We present a case of a premature girl born after 26 weeks of gestation who developed a Staphylococcus aureus sepsis with multiple Septic Arthritides (SA), Osteomyelitis (OM) of multiple joints and abscess formation in the gluteus maximus muscle on the right side. This was treated surgically and with antibiotics with good outcome.

\section{Clinical Signs and Pathogenesis}

Two percent of all OM during childhood consists of neonatal infections. Clinical signs are often nonspecific in the neonatal period in comparison to OM in childhood [2,3]. Signs described are vague like nutritional difficulties and generalized as well as localized edema, increased tenderness and erythematous skin. Pain sometimes limits movement of the involved extremity. Fever is also non-specific. Thus, clinical presentation can range from local complains to general complaints due to transient bacteremia and sepsis. Penetrating


MrR-otal body (left to right)
Sagittal I1 post gadolinium of the ankle shows a fluid collection with rim enhancement in the ankle. Tarsal bones are partially involved Sagittal T1 post gadolinium of the eltow shows
and enhancement of the bone. (dotted arrow)

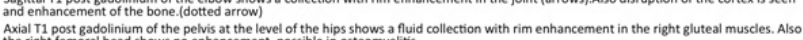

\section{L}

Figure 2: MRI-total body (left to right)

Sagittal T1 post gadolinium of the ankle shows a fluid collection with rim enhancement in the ankle. Tarsal bones are partially involved.

Sagittal T1 post gadolinium of the elbow shows a collection with rim enhancement in the joint (arrows). Also disruption of the cortex is seen and enhancement of the bone (dotted arrow).

Axial T1 post gadolinium of the pelvis at the level of the hips shows a fluid collection with rim enhancement in the right gluteal muscles. Also the right femoral head shows no enhancement, possible in osteomyelitis.

trauma, surgery, invasive procedures (such as umbilical artery catheterization) and prematurity are seen as potential risk factors for hematogenous spread of bacteria $[1,3]$. The strongest risk indicator for developing deep infections like an $\mathrm{OM}$ is a persisting positive blood culture. Blood cultures remain the golden standard for establishing the bacterial source of the OM. Because antibiotics often are already started when surgery is performed, cultures from the bone usually are sterile. However, only in half of the cases blood cultures are positive. If no clear focus or no positive blood culture is found, diagnostic imaging should be performed [2].

Staphylococcus aureus is a prominent pathogen in neonatal septicemia and carries a high risk of developing deep-seated infections such as OM [4]. Other commonly found pathogens are Streptococcus agalactiae, Escheria coli, Klebsiella and Enterobacter [1].

Multifocal OM is more frequently seen in neonates compared to older children during childhood and has been reported in $20-50 \%$ of all neonates with OM foci [2]. Lower extremities are significantly more often involved [5]. Localization of OM in neonates differs from older children due to a different blood supply to the bone of the neonate. The metaphysical vessels communicate with the epiphysial vessels of the cartilaginous precursor of the ossific nucleus. This facilitates a rapid spread of infections from the metaphysis to the epiphysis, which is often destroyed in the infection. This explains why in neonates arthritis and osteomyelitis co-exist [6]. Premature babies have an underdeveloped haemato-lymphoreticular system, which is required for fighting bacteremia. That might be the reason why bacteremia leads subsequently relatively easy to septic arthritis and osteomyelitis. The incidence in newborns is low $(0.5 \%$ of all neonatal admissions), especially among preterms [7]. Cases are described in term and late preterm newborns with presentation of symptoms from 2-53 days. Prematurity herein is not clearly defined. As far as we know, the case presented is the youngest patient in literature presenting with a multifocal OM [8].

A noteworthy in the presenting case is the relapse multifocal osteomyelitis and arthritides without positive pus cultures for the original pathogen, Staphylococcus aureus, of infection. Furthermore no pathogen was found. A clear notification for this phenomenon is 
not found. The diagnosis Chronic Recurrent Multifocal Osteomyelitis (CRMO) could be considered. CRMO is a chronic recurrent multifocal osteomyelitis of unknown noninfectious etiology [9]. Differs microorganisms including Staphylococci, Streptococci, Mycoplasma as well autoimmune process has been suggested but not yet confirmed. CRMO affects metaphysis of the long bones, pelvis, clavicle and mandible. Radiology X-ray and MRI can show similarities to a bacterial osteomyelitis [9]. Bone pains are usually the man symptoms; however this was difficult to report in our case of the premature neonate [10]. Increase of inflammatory markers can be seen in $66 \%$ of the case [11]. Though this phenomena is rare and mainly effects children in the age of 4-14 years [9].

\section{Diagnosis}

As described before, the presentation of OM in neonates is often by clinical presentation of joint swelling and a positive blood culture $[7,12]$. Laboratory findings other than blood cultures are variable and thought to be non-specific [2]. Increased sedimentation rate is seen in $90 \%$ and increased CRP in $98 \%$ of the cases. CRP is time-sensitive and can be used as an illness progression indicator. Moreover it can be used to monitor the effect of therapy. In the presented case, the CRP increased to $305 \mathrm{mg} / \mathrm{l}$ and then decreased during therapy to $1.2 \mathrm{mg} / \mathrm{l}$. The White Blood Cell count (WBC) is of little value in diagnosing neonatal OM [1]. However, in our case high levels of WBC count were found.

Radiology plays an important role in the early detection and treatment of OM. Usually conventional imaging is the first modality of choice. Conventional imaging may show early signs of osteomyelitis, but early signs of bone destruction may not be evident on X-ray until about 10 days after onset of symptoms. Thus, a normal x-ray cannot be used to exclude OM in the first 10 days after onset of symptoms. In young children, ultrasound is a useful modality to combine physical exam with radiologic findings. Ultrasound has a high sensitivity in detection of infiltration of soft tissue, (subperiosteal) abscess formation, fistulas or fluid in a joint early in the process. Disruption of the bone cortex can be seen. The main drawback of ultrasound is that bone marrow cannot be imaged reliably. MR has an important role in the detection of acute osteomyelitis. It has a high sensitivity for detection and allows imaging of bone marrow, soft tissue, subperiosteal and articular collections. Additionally, complications of osteomyelitis like abscess formation or arthritis can be seen on MR. The main drawback of MR is the long time of scanning, requiring immobilization of the patient. However, children of this age group can be fixated without requiring anesthesia per se. Swaddling after feeding often is enough. CT plays a limited role in the detection of OM. CT can be used to detect complications of osteomyelitis, such as sequester. The main drawback of CT is the high radiation dose and soft tissues are not well analyzed [13].

\section{Treatment}

Early recognition and treatment are essential for successful outcome [2]. Drainage of abscesses and arthritis should be initiated immediately, after which intravenous treatment should be started as soon as possible. Treatment involves optimal antibiotic regimen, pain management and if necessary, repeated surgical intervention $[1,14]$. Antibiotic treatment during 3-6 weeks is the primary treatment. Antibiotics should be specified for the outcome of the bacterial culture. In the presenting case the Staphylococcus aureus was sensible for flucloxacillin, however due to the progression and the severity of the infection rifampicin was started besides flucloxacilline. Because of the possible severity of sight effect of rifampicin, especially in this small premature neonate, we tried to limit the duration of treatment and it was chosen to continue the flucloxacillin.

Sequential parenteral-oral therapy may be used with close follow up. Shorter duration of parenteral therapy follow by oral therapy based on good clinical response was not associated with recurrence of infection [15]. If the clinical response to therapy is satisfactory, antibiotics can be continued orally. However no specific evidence based recommendations can be made and no specific time markers are described in the literature $[1,16]$. Peltola et al., propose a shorter treatment in patients older than 3 months [15]. Shorter duration of parenteral therapy followed by oral therapy based on good clinical response was not associated with recurrence of infection [12]. Since few reports of OM in newborns and premature newborns have been published no specific guidelines are available for premature babies suffering from multifocal osteomyelitis $[15,16]$. In the presented case, the antibiotic regime was continued for 6 weeks after the last increase of swelling and infection parameters in blood.

Surgical decompression and debridement was conducted in the presented case because of the presence of abscesses and multifocal septic arthritis and osteomyelitis. MR showed to be of significant value for pre-operative planning. Pre-operative localization of intra osseous abscesses was seen in advance and resulted in adequate drainage and shorter surgery time. Deshpande et al., reviewed 15 causes of neonatal septic arthritis and advised immediate arthrotomy to drain pus from the joint in case of strong clinical suspicion, instead of waiting $72 \mathrm{~h}$. This resulted in a better long-term outcome with less functional sequellae [7].

\section{Long Term Follow-Up}

Especially newborns with concomitant septic arthritis and osteomyelitis are at risk for long term functional sequellae [17]. Better long-term results can be achieved by early surgical drainage and immediate appropriate antibiotic coverage [1]. Multiple risk factors are associated with bad outcomes in the long term: delay in diagnosis or surgical drainage, involvement of hip or shoulder, short duration of antimicrobial therapy, high serum CRP and slow clinical response to therapy $[1,3]$.

\section{Conclusion}

We present a case of OM in a premature neonate of 26 weeks with favorable outcome. Recognition of OM can be difficult; an important sign is persisting positive blood cultures without any other infectious focus. We have the opinion that through physical examination in case of positive blood cultures and raised infectious parameters suspicion of OM should be raised. After initial imaging, additional imaging should be performed, preferably MR imaging. Radiology should always be used as confirmation of the clinical suspicion or diagnosis. In case there are abscesses, either in the soft tissues or intramedullary, these should be surgically drained. The same principle accounts for septic joints. Early recognition and aggressive treatment of $\mathrm{OM}$ is of paramount importance in preventing long-term sequelae.

\section{References}

1. Goldmann DA, Durbin WA Jr, Freeman J (1981) Nosocomial infections in a neonatal intensive care unit. J Infect Dis 144: 449-459.

2. Frederiksen B, Christiansen P, Knudsen FU (1993) Acute osteomyelitis and septic arthritis in the neonate, risk factors and outcome. Eur J Pediatr 152: 577-580. 
Citation: Somford MP, Huibers MHW, Schuppen J, Struijs PAA, van Lee R (2015) Multifocal Osteomyelitis in a Neonate, an Overview of Diagnosis and Treatment. J Orthop Res Physiother 1: 015.

3. Yagupsky P, Bar-Ziv Y, Howard CB, Dagan R (1995) Epidemiology, etiology, and clinical features of septic arthritis in children younger than 24 months. Arch Pediatr Adolesc Med 149: 537-540.

4. Ross AC, Toltzis P, O'Riordan MA, Millstein L, Sands T, et al. (2008) Frequency and risk factors for deep focus of infection in children with Staphylococcus aureus bacteremia. Pediatr Infect Dis J 27: 396-399.

5. Al Saadi MM, Al Zamil FA, Bokhary NA, Al Shamsan LA, Al Alola SA, et al. (2009) Acute septic arthritis in children. Pediatr Int 51: 377-380.

6. Ogden JA (1979) Pediatric osteomyelitis and septic arthritis: the pathology of neonatal disease. Yale J Biol Med 52: 423-448.

7. Deshpande SS, Taral N, Modi N, Singrakhia M (2004) Changing epidemiology of neonatal septic arthritis. J Orthop Surg (Hong Kong) 12: 10-13.

8. Berberian G, Firpo V, Soto A, Lopez Mañan J, Torroija C, et al. (2010) Osteoarthritis in the neonate: risk factors and outcome. Braz $\mathrm{J}$ Infect Dis 14 413-418.

9. Aygun D, Barut K, Camcioglu Y, Kasapcopur O (2015) Chronic recurrent multifocal osteomyelitis: a rare skeletal disorder. BMJ Case Reports.
10. Catalano-Pons C, Comte A, Wipff J, Quartier P, Faye A, et al. (2008) Clinical outcome in children with chronic recurrent multifocal osteomyelitis. Rheumatology (Oxford) 47: 1397-1399.

11. Brown T, Wilkinson RH (1988) Chronic recurrent multifocal osteomyelitis. Radiology 166: 493-496.

12. McPherson DM (2002) Osteomyelitis in the neonate. Neonatal Netw 21: 9-22.

13. van Schuppen J, van Doorn MM, van Rijn RR (2012) Childhood osteomyelitis: imaging characteristics. Insights Imaging 3: 519-533.

14. Korakaki E, Aligizakis A, Manoura A, Hatzidaki E, Saitakis E, et al. (2007) Methicillin-resistant Staphylococcus aureus osteomyelitis and septic arthritis in neonates: diagnosis and management. Jpn J Infect Dis 60: 129-131.

15. Peltola $H$, Pääkkönen $M$, Kallio $P$, Kallio $M J$, Osteomyelitis-Septic Arthritis (OM-SA) Study Group (2009) Prospective, randomized trial of 10 days versus 30 days of antimicrobial treatment, including a short-term course of parenteral therapy, for childhood septic arthritis. Clin Infect Dis 48: 1201-1210.

16. Caramia G, Ruffini E, Zaffanaello M, Fanos V (2007) Acute bone and joint infections in children and therapeutic options. J Pediatr Infect Dis 2: 193-203.

17. Vazquez M (2002) Osteomyelitis in children. Curr Opin Pediatr 14: 112-115. 